\title{
Dental Post-Mortem Computed Tomography for Disaster Victim Identification: A Literature Review
}

\author{
Elisa Nguyen ${ }^{1 *}$ and Edel Doyle ${ }^{2}$ \\ ${ }^{1}$ Department of Medical Imaging and Radiation Sciences, Monash University, Australia \\ ${ }^{2}$ Lake Imaging, Australia \\ *Corresponding author: Elisa Nguyen, Department of Medical Imaging and Radiation Sciences, Monash University, Clayton, Australia
}

Submission: March 28, 2018; Published: 㭗 September 17, 2018

\begin{abstract}
Over the last decade, post-mortem computed tomography (PMCT) has been increasingly adopted within the forensic setting. The three-dimensional (3D) capability and non-destructive approach of PMCT allows it to have several advantages over conventional x-rays, and as such it may be of potential use within forensic odontology for disaster victim identification (DVI). However, the implementation of dental PMCT can only have a real forensic benefit if its accuracy is comparable to that of conventional post-mortem (PM) dental radiographs; which is currently standard practice. Therefore, the purpose of this review was to evaluate if dental PMCT is as accurate as PM dental radiographs. A review of the current literature has shown the accuracy of dental PMCT to be comparable or like that of PM dental radiographs. As a result, there is a potential for dental PMCT to replace conventional PM dental radiographs in the DVI process of Mass Fatality Incidents (MFIs). However, further research is required to establish an international standard protocol for dental PMCT before it can be recognised as a new standard practice within the forensic setting.
\end{abstract}

Keywords: Forensic radiology; Post-mortem computed tomography; Disaster victim identification; Forensic odontology; Dental identification

\section{Introduction}

Forensic identification of the deceased is important not only for jurisdictional reasons but also reconciliation, so victims can be returned to their families [1]. However, this process can become complex in mass fatality incidents (MFIs) that are international in scope, such as terrorist attacks or natural disasters. Therefore, INTERPOL has devised a guidebook that promotes an international standard for disaster victim identification (DVI) [2].

Identification of individuals is typically achieved by comparison of available ante-mortem (AM) and post-mortem (PM) data. From this comparison, forensic experts can make a conclusion on the identity of the individual based on their level of confidence. The INTERPOL DVI guidebook highlights four different conclusions that can be made, which include: positive identification (AM and PM data are from the same individual), probable identification (some characteristics match but data minimal), possible identification (no exclusion can be made as data minimal) and excluded identity (AM and PM data are from different individuals) [2]. The methods utilised to assist in the identification of victims can be categorised as either primary or secondary. Primary methods of identification are considered the most reliable and classically consist of scientifically sound techniques. The three internationally recognised primary methods include: forensic odontology, fingerprints and DNA samples. In contrast, secondary means ordinarily do not provide enough information for a definitive identification, and so tend to be used in combination with primary methods to support or verify findings. Secondary identifiers include aspects such as: personal descriptions (for example gender and age), medical findings and clothing/evidence discovered on victims' bodies (for example jewellery or personal documents) [2].

Forensic odontology is one of the primary approaches to identification because dentition is unique to everyone. As human teeth are the hardest substances in the body, this makes them highly resilient and particularly useful in cases of burning or decomposition, where soft tissue structures of the body are no longer accessible [3]. In the PM setting, dental records are obtained through the combination of: radiographs, visual examination and dental charting [4]. Radiology is considered an important aspect of forensic dental identification. Dental radiography provides an objective anatomical overview compared to dental charts or written records, which can have honest errors or falsifications [5]. The classical dental radiographs obtained through conventional $\mathrm{x}$-rays can be categorised as intra-oral (periapical, bitewing and occlusal) or extra-oral (orthopantomogram/OPG) [6-8]. The image geometry of the PM dental radiographs should replicate those of the AM radiographs for effective comparison [9]. 
Full-body post-mortem or multi-slice computed tomography (PMCT or MSCT) has rapidly been adopted in forensic investigations and currently is recommended to have a role in DVI $[1,10-16]$. The three-dimensional (3D) capability of MSCT allows it to have several advantages over conventional x-rays. One of the main benefits is the ability to reconstruct and match the PMCT data to any AM radiologic image; such as dental radiographs [17]. This allows PMCT to become a potential tool for dental identification, particularly when it is not possible to replicate the image geometry of the PM and AM dental radiographs [18]. Additionally, in cases of burning, where teeth can become brittle after exposure to high temperatures, MSCT provides an ideal non-destructive approach compared to conventional x-rays; which may require handling of the oral cavity and further possible damage of brittle teeth $[19,20]$. However, the primary limitation in dental PMCT is the presence of highly radiopaque dental restorations, such as amalgam, which can cause streak artefacts [21]. This leads to problems with defining edges of teeth and fillings, and potentially compromises the comparison between dental PMCT and AM dental radiographs [22,23]. As a result, there has been a growing amount of literature that describes methods to reduce metal streak artefacts $[3,20,21,24]$.

Alternatively, there has been discussion for the use of cone beam computed tomography (CBCT) in forensic dentistry $[14,25,26]$. CBCT utilises a conical $x$-ray beam, where the fan angle extends in both $\mathrm{x} / \mathrm{y}$ and $\mathrm{z}$ directions and is centered on a two-dimensional (2D) detector panel. One rotation of the x-ray tube and detector allows greater coverage of the area of interest, thus allowing several hundreds of 2D images to be produced during this time [27]. Currently, CBCT systems can be classified into three different types (based on the positioning of the individual being scanned) which include: sitting, standing and supine [28]. The sitting and standing CBCT units are impractical for imaging deceased individuals as half heads/mandibles would need to be detached from the body before scanning [25]. This invasive disfigurement is not ethically permitted, and therefore it has been recommended that supine CBCT systems be utilised in forensic settings [25,29]. In these situations, the advantages of CBCT over MSCT include: less metal streak artefacts, shorter scan times, reduced cost and smaller CT units that are easier to transport to the disaster scene [25]. Nonetheless, CBCT lacks accuracy in Hounsfield Units and provides minimal information on surrounding soft tissues $[27,30]$. These are factors seen to assist in the differentiation of dental restorations and the forensic identification of victims $[3,31]$.

\section{Aim}

Overall, the implementation of dental PM computed tomography (CT) can only have a real benefit in forensic identifications if its accuracy is comparable to that of conventional PM dental radiographs; which is currently standard practice. This article presents a review of the literature to assess the accuracy of dental PMCT in comparison to PM dental radiographs, and hence could replace it in the DVI process of MFIs.

\section{Methods}

Searches were performed through: Ovid Medline, Science Direct and Scopus databases. The central themes of this review's aim were determined, and every search had a combination of key words relating to all central themes (Table 1). The key terms within each central theme were grouped together with an 'OR', whilst the central themes combined by 'AND'. An example of a search used was: [computed tomography OR "CT" OR "MDCT" OR cone beam* OR mobile*] AND [dent* OR t\#\#th OR oral* OR odont*] AND [radiograph* OR imaging OR xray*] AND [forensic* OR post mortem* OR identif*] AND [disaster* OR victim* OR incident* OR traged*]. The searches were limited to the English language. Publications before the year 2000 were excluded due to the limited use of CT in forensic imaging prior to this. Initial selection criteria included papers that addressed CT of the dentition in the context of forensic investigations. Additional secondary sources were found through citations or reference lists of relevant papers. After application of the eligibility criteria, a total number of 37 articles were found and analysed further.

Table 1: The search terms used for this literature review comprised of a varying combination of key words from all central themes

\begin{tabular}{|c|c|}
\hline \multirow{4}{*}{ Central Themes } & Key Words Used in Search \\
\hline \multirow{4}{*}{$\begin{array}{c}\text { Computed tomography } \\
\text { (CT) }\end{array}$} & -computed tomography OR “CT" \\
\cline { 2 - 3 } & -cone beam* \\
\cline { 2 - 3 } & -multi detector OR “MDCT" OR multi slice OR MSCT \\
\cline { 2 - 3 } & -virtual \\
\cline { 2 - 3 } & -mobile* \\
\cline { 2 - 3 } & -post mortem computed tomography OR PMCT \\
\hline
\end{tabular}




\begin{tabular}{|c|c|}
\hline \multirow{6}{*}{ Dentition } & -dent* OR dental OR dental identification \\
\hline & -t\#\#th OR teeth \\
\hline & -incisors \\
\hline & -molars \\
\hline & -oral* \\
\hline & -odont* OR odontology \\
\hline \multirow{7}{*}{ Dental x-rays } & -imaging* \\
\hline & -xray* \\
\hline & -radiograph* \\
\hline & -orthopantogra* OR panoramic \\
\hline & -periapical* \\
\hline & -bitewing* \\
\hline & -occlusal* \\
\hline \multirow{4}{*}{$\begin{array}{c}\text { Post-mortem (PM) \& } \\
\text { Forensics }\end{array}$} & -post mortem* \\
\hline & -forensic* OR forensic science \\
\hline & -anthropolog* \\
\hline & -identif* \\
\hline \multirow{8}{*}{$\begin{array}{l}\text { Disaster Victim Identi- } \\
\text { fication (DVI) process \& } \\
\text { Mass Fatality Incident } \\
\text { (MFI) }\end{array}$} & -disaster* \\
\hline & -victim* \\
\hline & -disaster victim identification OR DVI \\
\hline & -incident* \\
\hline & -traged* \\
\hline & -casualt* \\
\hline & -mass* \\
\hline & -fatal* \\
\hline
\end{tabular}

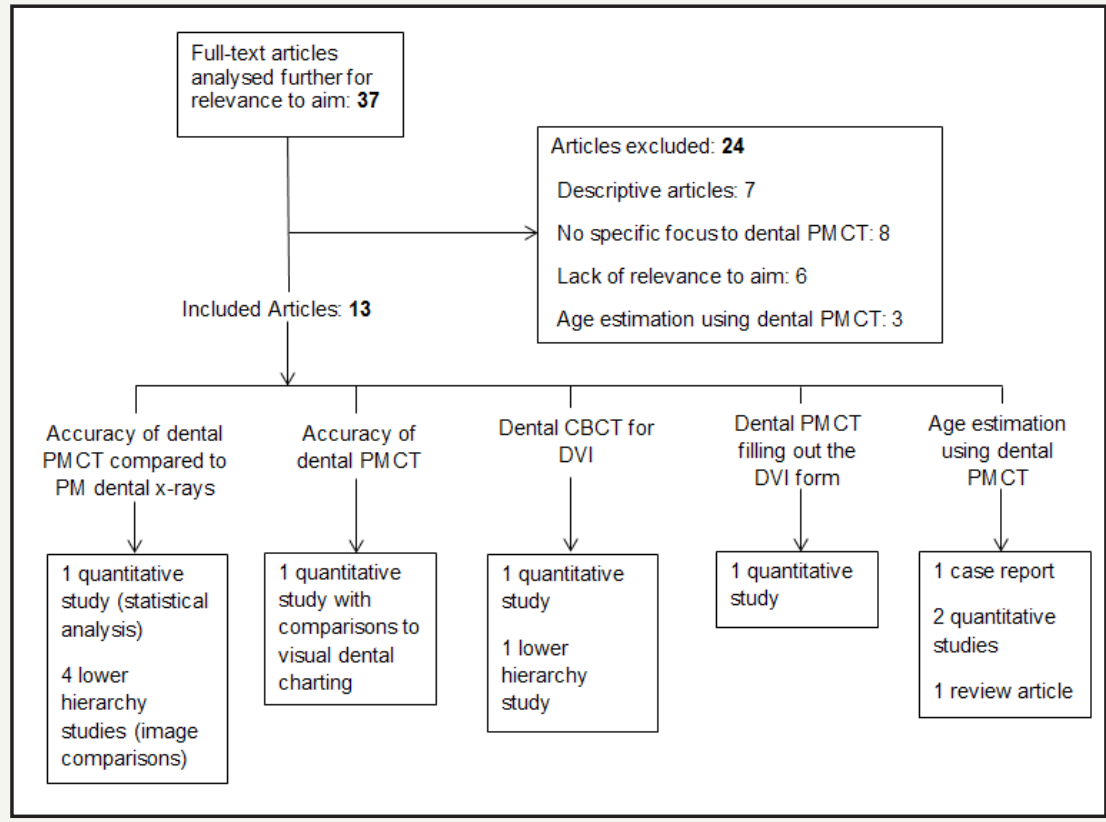

Figure 1: 


\section{Results}

Figure 1 of the 37 articles analysed, seven were considered as descriptive articles, meaning that they were review articles which just stated information without adequately referencing or evaluating original research. Three out of these seven descriptive articles did not use any evidence to support their claims, and so were omitted from the study sample $[1,17,32]$. The remaining four descriptive articles provided a brief overview of the current state of knowledge of dental PMCT, however did not go further to analyse original research, hence were also excluded $[8,23,27,33]$. Instead, these descriptive articles were utilised for general background reading. Eight articles had aims that were too broad and did not specifically focus on dental CT, hence were excluded [9,10,11$16,24]$. Six papers were excluded due to lack of relevance to the aim; as they were either focused on methods to reduce streak artefacts, discrimination of dental fillings with CT, matching software's for dental radiograph comparison or the use of AM laser surface scanning [3,20,21,34-36]. Seven articles were found to be related to age estimation using dental PMCT. Four out of these seven articles were included as they either demonstrated that this secondary anthropological technique could lead to DVI, or they compared dental PMCT to conventional dental radiographs as part of their study methodology [19,37-39]. The remaining three articles were excluded because they did not satisfy either condition $[15,40,41]$.

One article was included as it assessed the accuracy of dental PMCT in terms of filling out the DVI INTERPOL form [42]. Two articles were found to be related to the implementation of CBCT within forensic DVI; hence both were included within the study sample $[25,26]$. One article was found to address the quantitative accuracy of dental PMCT by comparing it to visual dental charting. Even though the study did not make comparisons to conventional PM dental radiographs, it was still included as it addressed the subaim of the accuracy of dental PMCT for DVI [22].

Four articles that were relevant to the aim were included but were classified as lower hierarchy evidence $[6,18,43,44]$. This was because the articles only conducted subjective visual image comparisons between dental PMCT and dental radiographs without going into detail about the concordant points they used to compare each tooth. Additionally, no statistical analysis was performed to assess the accuracy of dental PMCT. For dental PMCT to be implemented in practice for DVI, qualitative research was not considered scientifically sound evidence to be presented in court.

Therefore, only one academically rigorous article was found to address the aim through appropriate quantitative analysis [31]. This resulted in a total of 13 inclusions and 24 exclusions.

\section{Discussion}

\section{Potential value/contribution of dental PMCT for DVI}

The potential use of PMCT within forensic odontology for DVI has been highlighted throughout literature; including the INTERPOL
DVI guidebook $[2,6,43,44]$. The basis of this idea stems from the fact that PMCT data can be reconstructed to mimic any AM dental radiograph for comparison. This becomes particularly useful when difficulty is faced with matching the x-ray beam geometry between conventional PM and AM dental radiographs [18]. Conversely, in cases where the AM data is not available during the time of the scan, the PMCT data can be reconstructed later without having to rescan the body Thali et al. [17]. conducted one of the first studies to test the feasibility of dental CT processing software (Dentascan) in the forensic setting, demonstrating that panoramic-like images could be reconstructed for all putrefied corpses involved in the study [43]. Other relevant articles show similar findings however the algorithm or method utilised to reconstruct the PMCT data is seen to vary across studies. For example, Jackowski et al. [44] \& Tohnak et al. [6]. establish the reconstruction ability of CT through a different algorithm based on the maximum intensity projection (MIP). The variation observed in the CT reconstruction method demonstrates the lack of an international standard protocol for dental PMCT. This is reflected through the INTERPOL DVI guidebook which does not have a specific section for PMCT [1].

A range of research has been conducted to assess the degree to which dental MSCT can be used to fill out the PM INTERPOL form for DVI $[10,42]$. A study by Sidler et al. [10] found that PMCT could fill out the majority of section D2-45 on the INTERPOL DVI form, which consists of the forensic pathologist briefly describing the condition of a victim's teeth [10]. Nonetheless, in the case of dentures where an identification number was required, a physical examination was still conducted as PMCT could not reveal this information. The study went on further to highlight that dental PMCT could be used to fill out section F of the DVI form; which requires the forensic odontologist to provide more extensive descriptions of dental findings. However, did not specifically focus on section F of the DVI form as part of their study, and so it was unclear as to the extent to which this section could be completed using dental PMCT. In saying so, Franco et al. [42] conducted a study that verified which sections of the PM F1 and F2 INTERPOL forms for DVI could be completed using dental PMCT.

They found that PMCT could not differentiate between different compositions of dental restorations, which formed a moderate percentage (33\%) of the F1 and F2 INTERPOL sections [42]. As a result, it may be seen that a forensic odontologist would still be required to perform a physical dental examination within these cases. Nevertheless, when only focusing on the sections of the F1 and F2 INTERPOL forms that could be filled out using dental PMCT, Franco et al. [42] highlighted that an accuracy of $82.6 \%$ was obtained. Additionally, both studies by Sidler et al. [10]. Did not address the extent to which conventional dental radiographs could fill out the PM INTERPOL form $[10,42]$. Therefore, no comparisons could be made between dental PMCT and PM dental radiographs in terms of which could fill out a greater proportion of the INTERPOL DVI form. 
Tohnak et al. [6]. proposed that it was feasible to replace conventional PM dental radiographs with dental PMCT for forensic identification. However, this statement was deemed invalid as no analysis was conducted to assess whether identification was possible from the CT data produced. Nonetheless, the studies by Thali et al. \& Jackowski et al. $[17,20]$ both demonstrated that positive identification was achievable through the comparison of dental PMCT and AM dental radiographs [43,44]. Similarly, both studies utilised the same retrospective case of a single putrefied corpse from the Virtopsy project.

Therefore, even though different reconstruction methods were utilised in each study, identification was still possible in both instances. Nevertheless, the study by Jackowski et al. [20] had a sample size that only consisted of that one putrefied corpse, and thus their results may not be generalisable to all forensic cases for DVI [44]. In contrast, the study further examined two additional cases and emphasised that positive identification with PMCT was viable when corpses had unique dental features present [43]. Therefore, in cases with relatively common dental features, conventional PM dental radiographs were still required to confirm a positive identification; even when the PMCT images were highly indicative of the assumed victim. In saying so, this study did not give any descriptions on the individuals who analysed the images, including how experienced they were with dental identification using PMCT. Overall, these studies were deemed as qualitative in nature as they evaluated dental PMCT for victim identification through visual image comparisons. Consequently, the lack of quantitative statistical analysis makes it difficult to compare the accuracy between dental PMCT and conventional PM dental radiographs. Therefore, these articles are insufficient to justify whether dental PMCT should replace standard PM dental radiographs in the forensic DVI process.

\section{Accuracy of dental PMCT for DVI}

The use of conventional PM dental radiographs is recommended by INTERPOL and is currently an established tool within the DVI process [2]. The main reasons for this are because PM dental radiographs are considered relatively inexpensive, quick, efficient and accurate [45]. Throughout literature, studies have highlighted an accuracy that ranges between $85.5 \%$ and $93 \%$ for comparative identification through conventional PM dental radiographs; with one study showing a mean sensitivity and specificity of $89 \%$ and $96.7 \%$ respectively $[31,46,47]$.

Currently, the quantitative statistical accuracy of dental PMCT for DVI is sparse but has been explored in two main studies Kirchhoff et al. [22]. One study by compared dental PMCT to PM visual dental charting (instead of PM dental radiographs) and thus was considered to address a sub-aim of this literature review. In saying so, the quantitative results from this study may be extracted for information on the accuracy of dental PMCT. Out of a sample size of ten skulls, the study found that dental PMCT had a mean sensitivity and specificity of $88.2 \%$ and $97.1 \%$ respectively. The quantitative accuracy value was not explicitly stated within their study but could still be calculated as $95.3 \%$ based on the true positive/negative and false positive/negative results. When directly comparing this value to the accuracy previously mentioned for conventional PM dental radiographs, it appears that dental PMCT has a higher accuracy in terms of identifying the deceased. Nevertheless, this direct comparison may be invalid as each accuracy value was determined utilising different methodologies. The accuracy result within the study by [22] represented the ability of dental PMCT in describing the presence of dental fillings and their exact locations [22]. Therefore, their study demonstrated that CT was associated with error rates; as the presence of streak artefacts had the potential to cause interpretation errors. However, the study did not actually assess the accuracy of dental PMCT in terms of positive victim identifications. Therefore, a conclusion could not be made on whether dental PMCT could replace conventional PM dental radiographs for DVI.

In contrast, a study by Ruder et al. [31] was able to adequately assess the accuracy of dental PMCT in terms of correctly identifying deceased victims within a simulated mass fatality scenario. This was achieved by matching or excluding a total of 115 dental PMCT images and 114 AM bitewing radiographs; amongst which were 98 matching pairs. The mean accuracy for the correctly matched pairs (positive comparative identification) and correctly excluded images was found to be $92 \%$ and $80 \%$ respectively. The overall mean accuracy (proportion of all correct results) was not stated within the study by Ruder et al. [31]. Nonetheless, readers can calculate this value as $89 \%$ through the true positive/negative and false positive/ negative results listed within the study. This accuracy value (89\%) is seen to be like the accuracy of conventional PM dental radiographs for DVI (85.5\% to 93\%). As a consequence, comparing AM dental radiographs with dental PMCT appears to be just as reliable as comparing AM and PM dental radiographs for identification of deceased bodies. Therefore, the study found that the accuracy of comparative identification using dental PMCT was not significantly affected by the presence of streak artefacts (from dental fillings). In addition, the study by [31]. suffered from limitations where the raters were restricted to a single volume rendered image that had to be analysed on print-outs (instead of digitally on the computer). These aspects were likely to underestimate the accuracy of dental PMCT for victim identification; as forensic experts should have digital access to full datasets in real scenarios. Therefore, the accuracy of dental PMCT could be superior to that of conventional PM dental radiographs for DVI, however further research would be required to clarify this idea. Overall, these findings support the various institutions that have already routinely adopted dental PMCT within forensic victim identification [31].

\section{Influence of reviewer's experience on accuracy}

It has been found that the experience of assessors is an aspect that affects the accuracy of forensic identification [22,31]. There are two kinds of experience which appear necessary for successful identification through dental PMCT. The first consideration is assessor experience in relation to dental identification. It has 
been highlighted that assessors with practical experience in forensic dental identification perform better than those with little or no practical experience in this field [31]. This is demonstrated in the study [31], where three assessors experienced in dental identification (forensic radiologist, forensic pathologist and forensic odontologist) achieved a greater number of correct matches compared to two other assessors with no experience in dental identification (clinical radiologist and medical student) As a result, this emphasises the importance of assessors to have practical experience in forensic dental identification, in order to achieve accurate positive matches [31].

The second consideration is the assessor's experience in forensic radiology or specifically the imaging modality used to assist identification; which in this case is dental PMCT. The study highlighted that the quality of results was able to improve with time as assessors were exposed to more dental CT images. Additionally, the study found a forensic odontologist (experienced in dental identification but little experience with dental PMCT) to have a greater amount of false positive matches compared to those of a forensic radiologist and forensic pathologist (who were experienced in both dental identification and PMCT) [31]. This indicates the inverse relationship between the experience of assessors with dental PMCT and the amount of false positive matches obtained. As a consequence, victim identification through dental PMCT should only be performed by professionals highly experienced in both dental identification and PMCT. Currently, forensic pathologists appear to achieve more accurate results in relation to victim identifications through dental PMCT. This was demonstrated in a study where a forensic pathologist was able to achieve higher accuracy when compared to a forensic radiologist (who had the same experience with PMCT and dental identification). This finding was also found by Kirchhoff et al. [26] however it was unclear how much experience each assessor had in relation to forensic dental ID within this study [22]. There is yet to be research that compares the accuracy results obtained from a forensic odontologist and a forensic pathologist; where both have the same experience with PMCT and dental identification.

\section{Value/contribution of dental CBCT for DVI}

Limitations have been observed with MSCT in forensic dental identifications, specifically in regard to the occurrence of streak artefacts that are produced through beam hardening from metallic dental restorations. Initially, to try and overcome this limitation, a range of research was conducted to find methods that could suppress streak artefacts on MSCT images [3,20,21]. One example includes the 2006 study by Jackowski et al. [43] who proposed that streak artefacts could be reduced by performing reconstructions with an extended CT scale. Nonetheless, this study only tested the feasibility of this technique on single extracted teeth (with dental fillings) instead of entire dentitions. Fortunately, a study made use of the extended CT scale when reconstructing images of skulls with entire dentitions [22]. However, despite adoption of this technique, their study found that streak artefacts were still significantly evident on the MSCT images. As a result, further research is required to develop a method that can effectively suppress streak artefacts (on MSCT images) in the setting of full dentitions.

Alternatively, it has been found that dental images produced from CBCT are associated with reduced streak artefacts when compared to dental images produced from MSCT [30]. This has been established in clinical dental practice, where CBCT is currently preferred over MSCT for a wide range of dental applications $[25,27,30]$. Therefore, several authors have considered the use of CBCT within forensic odontology. Just like MSCT, CBCT can reconstruct images to match any conventional dental radiograph. This was demonstrated in the forensic setting by Trochesset et al. [25]. who introduced a CBCT reformatting technique through imaging cadaver half heads. Even though the study utilised a sitting CBCT unit, the authors were still able to establish the feasibility of making comparisons between PM dental CBCT images and AM dental radiographs for identification purposes.

\section{Accuracy of dental CBCT for DVI}

A study by Murphy et al. [26] went on further to explore the accuracy of CBCT for forensic dental identification. The study found CBCT to have an overall mean sensitivity and specificity of $96.6 \%$ and $98.4 \%$ respectively. The overall mean accuracy was calculated from the results of the study and found to be $98.1 \%$. The authors claimed that the analysis method employed in their study was like that utilised [22] (who assessed the accuracy of dental MSCT) and concluded that their results were favourable when compared to the accuracy values of MSCT [26]. However, this appeared misleading as the gold standards utilised within each study were found to be different. used conventional panoramic radiographs as their gold standard, whilst utilised visual dental charting. Therefore, it may be invalid to directly compare the accuracy results of CBCT (obtained by Murphy et al. [26] to the MSCT accuracy results (obtained by Kirchhoff et al. [22]. Therefore, more studies are required in this area to reliably compare the accuracy between CBCT and MSCT in relation to forensic dental identifications.

By taking the results from the conventional panoramic radiographs as gold standard, was able to demonstrate that dental CBCT had a similar accuracy to standard dental radiographs. However, the images utilised within this study were obtained retrospectively from patients who were alive during the time of the scan. Therefore, the dental CBCT images were of optimal quality as the subjects would have been positioned ideally within the scanner. In the forensic setting, the positioning of deceased individuals may be more challenging due to the risk of disfigurement or damage to the body, and as such an optimal image quality cannot be guaranteed [29]. Additionally, the studies that advocate the use of CBCT within forensic odontology have been found to utilise sitting or standing CBCT units. The study by Trochesset et al. [25] demonstrated that sitting CВCT units may be applicable for use with cadaver half heads or mandibles. However, in the case of intact bodies, it 
is not ethically acceptable to detach body parts for identification purposes [29]. Therefore, a sitting or standing CBCT is unlikely to be practical for use in forensic identifications. Nonetheless, authors have recommended the use of supine CBCT units (that are like MSCT systems), highlighting that these units are more suitable for forensic situations $[22,25]$. Therefore, further research is required to test the feasibility of supine CBCT units for dental identifications within a forensic scenario.

\section{Contribution of dental PMCT to secondary methods of DVI}

Estimating the age of deceased victims assists in building a biological profile, which can assist in forensic investigations. Age estimation can be determined through the assessment of either skeletal or dental development [37]. However, it has been outlined that age estimations made through assessments of dental development are more closely correlated to the chronological age of victims (when compared to age estimations made through assessments of skeletal development) as the dentition is not as greatly affected by the environment [39]. Dental age estimation methods are considered a secondary identification technique, which usually does not contribute individually to definitive identifications of disaster victims. In saying so, within certain situations such as closed disasters (where the number of deceased victims is known to belong to an identifiable group of individuals), dental age estimation may still contribute to a positive identification $[2,19]$. This is particularly significant for cases involving skeletal juvenile remains, where primary methods of identification within forensic odontology may not be applicable due to the reduced amount of AM dental radiographs taken within early childhood years. Additionally, other anthropological factors such as gender or ethnicity cannot be accurately evaluated until after puberty, thus making dental age estimation an important factor for identification of deceased juvenile victims.

Typically, PM dental radiographs are used to assist in the assessment of dental development for age estimation purposes. However, a range of authors have advocated for standard x-rays to be replaced with PMCT for dental age estimations to identify bodies [15,19,37-40]. This was established by Bassed and Hill, who presented a case report where PMCT was utilised for dental age estimation to discriminate between two juvenile victims of the Black Saturday bushfires. In this closed disaster, the extensive incineration of remains made the use of conventional radiography challenging and time consuming; as a great deal of delicacy was required to manipulate the head and place the image receptor within the victim's mouth. Therefore, the use of PMCT was preferable as it allowed a non-invasive approach that was able to preserve dental structures. Furthermore, just like PM dental radiographs, Based and Hill [19] found that it was feasible to match the reconstructed CT data against atlases of dental development; which allowed age estimations to be made based on the expected maturation of the dentition at each year of an individual's life from childhood to adulthood [39]. As a result, they concluded that PMCT was a reliable tool for assessing dental development for age estimation purposes [19].

Studies have been conducted to compare the agreement between PMCT and PM conventional x-rays in regard to dental age estimations of juvenile remains $[37,38]$. Most studies have highlighted that dental PMCT is directly comparable to conventional dental radiographs when used to estimate the age of deceased children. A study conducted by Brough et al. [32] compared age estimations obtained from both OPG radiographs and dental PMCT images of the same group of 19 individuals. The results of their study found that dental age estimations made through PMCT images were in excellent agreement to those obtained from corresponding conventional OPG radiographs; which was highlighted through an interclass correlation coefficient of 0.95 . In the same way, a study by Bassed et al. [37] concluded a similar finding for a group of 12 deceased individuals, however their results recorded a good kappa agreement score of 0.780 [37]. Nevertheless, the discrepancies observed were explained through dental root growths occurring between the times the OPG radiographs and CT scans were taken, thus resulting in different age estimations noted between the OPG and CT images of the same individual. Additionally, highlighted that the superimposition of structures commonly observed with conventional dental radiographs made it difficult to assess dental root apices for age estimation. Therefore, the reconstructed PMCT images made evaluation of dental development easier in these cases as superimposition was overcome through the assessment of all planes [38]. As a result, it appears viable to replace conventional PM x-rays with PMCT for dental age estimations of juvenile remains for secondary identification.

\section{Conclusion}

This article presented a review of current literature to assess the accuracy of dental PMCT in comparison to PM dental radiographs to accurately identify disaster victims. Most articles highlight PMCT as a valuable non-invasive tool and thus support its use for dental identification purposes. However, the main limitation that has prevented the adoption of dental MSCT within forensic practice is the presence of streak artefacts on images. Nevertheless, despite these artefacts, recent findings have shown the accuracy of dental MSCT to still be comparable or like that of PM dental radiographs. As a result, there is a potential for dental PMCT to replace conventional PM dental radiographs within the DVI process. Currently, there is no international standard protocol for dental PMCT, and as such further research should be conducted to establish this if PMCT is to be adopted as a new standard practice. In this way, the INTERPOL DVI guidebook can be updated to include a specific section for PMCT. On the other hand, СВCT has been introduced as an alternative to MSCT for dental identifications due to its ability to have reduced streak artefacts. In saying so, further research is still required to determine the feasibility and accuracy of supine CBCT units within a forensic scenario. Furthermore, 
the experience of observers regarding both the imaging modality and dental identification can affect the accuracy of results, and so forensic identifications through dental PMCT should only be performed by highly experienced experts.

\section{References}

1. Brough AL, Morgan B, Rutty GN (2015) Postmortem computed tomography (PMCT) and disaster victim identification. Radiol Med 120(9): 866-873.

2. http://www.interpol.int/INTERPOL-expertise/Forensics/DVI-Pages/ DVI-guide

3. Jackowski C, Wyss M, Persson A, Classens M, Thali MJ, et al. (2008) Ultra-high-resolution dual-source CT for forensic dental visualizationdiscrimination of ceramic and composite fillings. Int J Legal Med 122(4): 301-307.

4. Pretty IA, Sweet D (2001) A look at forensic dentistry-Part 1: The role of teeth in the determination of human identity. Br Dent J 190(7): 359-366.

5. Wood RE (2006) Forensic aspects of maxillofacial radiology. Forensic Sci Int 159(1): S47-S55.

6. Tohnak S, Mehnert AJ, Mahoney M, Crozier S (2007) Synthesizing dental radiographs for human identification. J Dent Res 86(11): 1057-1062.

7. Viner MD, Alminyah A, Apostol M, Brough A, Develter W, et al. (2015) Use of radiography and fluoroscopy in Disaster Victim Identification: Positional statement of the members of the Disaster Victim Identification working group of the International Society of Forensic Radiology and Imaging. J Forens Radiol Imag 3(2): 141-145.

8. Jeffery AJ (2010). The role of computed tomography in adult postmortem examinations: an overview. Diagn Histopathol 16(12): 546-551.

9. Taylor PT, Wilson ME, Lyons TJ (2002) Forensic odontology lessons: multishooting incident at Port Arthur, Tasmania. Forensic Sci Int 130(2): 174-182.

10. Sidler M, Jackowski C, Dirnhofer R, Vock P, et al. (2007) Use of multislice computed tomography in disaster victim identification-Advantages and limitations. Forensic Sci Int 169(2): 118-128.

11. Dedouit F, Telmon N, Guilbeau Frugier C, Gainza D, Otal P, et al. (2007) Virtual autopsy and forensic identification-practical application: a report of one case. J Forensic Sci 52(4): 960-964.

12. Persson A, Jackowski C, Engström E, Zachrisson H (2008) Advances of dual source, dual-energy imaging in postmortem CT. Eur J Radiol 68(3): 446-455.

13. Steffen R, Suter C, Patricia F, Lars O, Michael T, et al. (2010) Multi-slice computed tomography (MSCT) of mountaineering casualties in the Swiss Alps-Advantages and limitations. Legal Med 12(6): 271-275.

14. Odonnell C, Iino M, Mansharan K, Leditscke J, Woodford N (2011) Contribution of postmortem multidetector CT scanning to identification of the deceased in a mass disaster: Experience gained from the 2009 Victorian bushfires. Forensic Sci Int 205(1): 15-28

15. Sakurai T, Michiue T, Ishikawa T, Yoshida C, Sakoda S, et al. (2012) Postmortem CT investigation of skeletal and dental maturation of the fetuses and newborn infants: a serial case study. Forensic Sci Med Pathol 8(4): 351-357.

16. Rutty GN, Robinson C, Morgan B, Black S, Adams C, et al. (2009) Fimag: The United kingdom disaster victim/forensic identification imaging system. J Forensic Sci 54(6): 1438-1442.

17. Hatch GM, Dedouit F, Christensen AM, Thali MJ, Ruder TD (2014) RADid: A pictorial review of radiologic identification using postmortem CT. J Forens Radiol Imag 2(2): 52-59.
18. Birngruber CG, Obert M, Ramsthaler F, Kreutz K, Verhoff MA (2011) Comparative dental radiographic identification using flat panel CT. Forensic Sci Int 209(1): e31-e34.

19. Bassed RB, Hill AJ (2011) The use of computed tomography (CT) to estimate age in the 2009 Victorian Bushfire Victims: A case report. Forensic Sci Int 205(1): 48-51.

20. Woisetschläger M, Lussi A, Persson A, Jackowski C (2011) Fire victim identification by post-mortem dental CT: Radiologic evaluation of restorative materials after exposure to high temperatures. Eur J Radiol 80(2): 432-440

21. Jackowski C, Lussi A, Classens M, Kilchoer T, Bolliger S, et al. (2006) Extended CT scale overcomes restoration caused streak artifacts for dental identification in CT--3D color encoded automatic discrimination of dental restorations. J Comput Assist Tomogr 30(3): 510-513.

22. Kirchhoff S, Fischer F, Lindemaier G, Herzog P, Kirchhoff C, et al. (2008) Is post-mortem CT of the dentition adequate for correct forensic identification?: comparison of dental computed tomograpy and visual dental record. Int J Legal Med 122(6): 471-479.

23. Dedouit F, Savall F, Mokrane FZ, Rousseau H, Crubézy E, et al. (2014) Virtual anthropology and forensic identification using multidetector CT. Br J Radiol 87(1036): 1-12.

24. Beck JJ (2011) What is the future of imaging in forensic practice? Radiography. 17(3): 212-217.

25. Trochesset DA, Serchuk RB, Colosi DC (2014) Generation of intra-orallike images from cone beam computed tomography volumes for dental forensic image comparison. J Forensic Sci 59(2): 510-513.

26. Murphy M, Drage N, Carabott R, Adams C (2012) Accuracy and reliability of cone beam computed tomography of the jaws for comparative forensic identification: a preliminary study. J Forensic Sci 57(4): 964-968.

27. Alamri HM, Sadrameli M, Alshalhoob A, Sadrameli M, Alshehri MA (2012) Applications of CBCT in dental practice: A reivew of the literature. Gen Dent 60(5): 390-400

28. Pauwels R, Araki K, Siewerdsen JH, Thongvigitmanee SS (2015) Technical aspects of dental CBCT: state of the art. Dentomaxillofacial Radiol 44(1): 1-20.

29. http://www.integer.co.uk/resources/Clarke\%20Report_2001.pdf

30. Kaeppler G (2010) Applications of cone beam computed tomography in dental and oral medicine. Int J Comput Dent 13(3): 203-219.

31. Ruder TD, Thali YA, Rashid SNA, Mund MT, Thali MJ, et al. (2016) Validation of post mortem dental CT for disaster victim identification. J Forens Radiol Imag 5(1): 25-30.

32. Brough AL, Morgan B, Rutty GN (2015) The basics of disaster victim identification. J Forens Radiol Imag 3(1): 29-37.

33. Rutty GN, Brough A, Biggs MJ, Robinson C, Lawes SD, et al. (2013) The role of micro-computed tomography in forensic investigations. Forensic Sci Int 225(1): 60-66.

34. Abduo J, Bennamoun M (2013) Three-dimensional Image Registration as a Tool for Forensic Odontology. Am J Forensic Med Pathol 34(3): 260266.

35. Tohnak S, Mehnert A, Mahoney M, Crozier S (2009) Dental identification system based on unwrapped CT images. Conf Proc IEEE Eng Med Biol Soc 2009: 3549-352.

36. Sakuma A, Makino Y, Saitoh H, Chiba F, Ishii N, et al. (2015) Evaluation of a personal identification method using the fusion funciton of CT images and dental radiographs. Dentomaxillofacial Radiol 44(2):1-6

37. Bassed RB, Briggs C, Drummer OH (2011) Age estimation and the developing third molar tooth: an analysis of an australian population using computed tomography. J Forensic Sci 56(5): 1185-1191. 
38. Brough AL, Morgan B, Black S, Adams C, Rutty GN (2014) Postmortem computed tomography age assessment of juvenile dentition: comparison against traditional OPT assessment. Int J Legal Med 128(4): 653-638.

39. Brough AL, Rutty GN, Black S, Morgan B (2012) Post-mortem computed tomography and 3D imaging: anthropological applications for juvenile remains. Forensic Sci Med Pathol 8(3): 270-279.

40. Graham JP, Odonnell CJ, Craig PJ, Walker GL, Hill AJ, et al. (2010) The application of computerized tomography (CT) to the dental ageing of children and adolescents. Forensic Sci Int 195(1): 58-62.

41. Pinchi V, Pradella F, Buti J, Baldinotti C, Focardi M, et al. (2015) A new age estimation procedure based on the 3D CBCT study of the pulp cavity and hard tissues of the teeth for forensic purposes: A pilot study. J Forensic Leg Med 36(1): 150-157.

42. Franco A, Thevissen P, Coudyzer W, Develter W, Van De Voorde W, et al. (2013) Feasibility and validation of virtual autopsy for dental identification using the Interpol dental codes. J Forensic Leg Med 20(1): 248-254.
43. Thali MJ, Markwalder T, Jackowski C, Sonnenschein M, Dirnhofer R (2006) Dental CT imaging as a screening tool for dental Profiling: advantages and limitations. J Forensic Sci 51(1): 113-119.

44. Jackowski C, Aghayev E, Sonnenschein M, Dirnhofer R, Thali MJ (2005) Maximum intensity projection of cranial computed tomography data for dental identification. Int J Legal Med 120(3): 165-167.

45. Martin-de las Heras S, Valenzuela A, Villanueva E, Marques T, Exposito N, Bohoyo J (1999) Methods for identification of 28 burn victims following a 1996 bus accident in spain. J Forensic Sci 44(2): 428-431.

46. Walsh M, Reeves P, Scott S (2004) When disaster strikes; the role of the forensic radiographer. Radiography 10(1): 33-43.

47. Maclean DF, Kogan SL, Slitt LW (1994) Validation of dental radiographs for human identification. J Forensic Sci 39(5): 1195-200.
Creative Commons Attribution 4.0 International License

For possible submissions Click Here

\section{Submit Article}

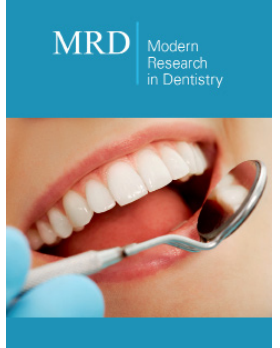

\section{Modern Research in Dentistry}

Benefits of Publishing with us

- High-level peer review and editorial services

- Freely accessible online immediately upon publication

- Authors retain the copyright to their work

- Licensing it under a Creative Commons license

- Visibility through different online platforms 